\title{
Endovascular Management of Acute Proximal Internal Carotid Artery Occlusions: The JHN Experience
}

\author{
Richard Dalyai, MD', Vismay Thakkar, BA², Saurabh Singhal, BA², Thana \\ Theofanis $^{3}$, BA Pascal Jabbour, MD¹, L. Fernando Gonzalez, MD', Aaron \\ Dumont, MD1, Robert Rosenwasser, MD1, Stavropoula I. Tjoumakaris, MD \\ 'Department of Neurological Surgery, Thomas Jefferson University, Philadelphia, PA \\ ${ }^{2}$ Visiting medical students, SMT. NHL Medical College, Ahmedabad, Gujarat, India \\ ${ }^{3}$ Jefferson Medical College, Thomas Jefferson University, Philadelphia, PA
}

\section{Introduction}

Stroke is a major cause of serious, long-term disability and the third leading cause of death, accounting for one in every 18 deaths in the United States. Approximately 800,000 strokes occur in the United States each year, leading to an estimated cost of 74 billion dollars in 2010. The severity and prognosis of patients with an acute internal carotid artery (ICA) occlusion is extremely poor. Studies have shown that $16-55 \%$ of patients will die from complications related to the infarction, $40-69 \%$ will be left with a profound deficit, and only $2-12 \%$ will make a reasonable recovery ${ }^{2}$. In young patients, internal carotid artery dissections presenting as an acute occlusion or thrombotic clot are responsible for approximately $14-20 \%$ of ischemic strokes. ${ }^{1}$ Results from trials utilizing emergent open surgical carotid recanalization or IVtPA have not been encouraging ${ }^{2,3}$. Recently, there have been small case reports of endovascular stent-assisted thrombolysis as a treatment option for patients with carotid occlusions and near occlusions. ${ }^{4-8}$

\section{Treatment Paradigm}

At Jefferson Hospital for Neuroscience, we have developed an aggressive treatment protocol for patients with acute internal carotid occlusions. On admission, all patients are assessed by the NIH Stroke Scale (NIHSS). Prior to endovascular intervention, a non-contrast head CT is performed as a pre-operative baseline. In addition, CT angiography and CT perfusion studies are obtained emergently. The decision to proceed with endovascular thrombolysis is based on clinical and radiographic analysis of pre-operative data. In summary, a minimum NIHSS of 6, CT perfusion with ischemic penumbra on mean transient time and cerebral blood flow but preservation of cerebral blood volume, and a detectable arterial occlusion on CT angiography are inclusion criteria for patient intervention.

All procedures are performed under general endotracheal anesthesia. Digital subtraction angiography is obtained focusing on the affected carotid circulation. An 8 French femoral sheath and 8 French guide catheter are used in most cases. An SL-10 and Transcend wire is used to cross the carotid bifurcation and determine the extent of the carotid occlusion. At that time, the decision is made for balloon angioplasty and carotid stenting. Balloon angioplasty is performed with an AVIATOR ${ }^{\mathrm{TM}}$ or Viatrac $^{\mathrm{TM}}$ balloon across the occluded segment and a Precise ${ }^{\circ}$ or ACCULINK ${ }^{\mathrm{TM}}$ stent is then deployed across the occluded internal carotid segment to the ipsilateral common carotid artery. Once the carotid artery is opened, angiography is performed to assess the intracerebral circulation. If a cutoff is still seen in the middle cerebral artery, further thrombolytic methods are utilized such as balloon angioplasty (Hyperglide ${ }^{\mathrm{TM}}$ balloon), Merci ${ }^{\oplus}$ or Penumbra ${ }^{\oplus}$ systems for aspiration and mechanical thrombolysis.

If mechanical thrombolysis is unsuccessful, and ictus is within 8 hours, pharmacologic thrombolysis is performed with intra-arterial tPA or ReoPro ${ }^{\circ}$. Finally, in all patients final angiography is performed to assess patency of the carotid circulation.

\section{Discussion}

The prognosis of acute ischemic stroke in the setting of a carotid occlusion or near occlusion has been shown to be extremely poor. The use of IV tPA has been the standard of care to date for patients with ischemic stroke presenting within a 4.5 hour time window from symptom onset. In the general setting of acute ischemic stroke, complete recanalization is achieved in only $30-40 \%$ of patients and less than $50 \%$ of patients on long term follow-up. In our study of acute carotid occlusions, these outcomes may be even less encouraging. Studies comparing the recanalization rates with IV TPA for acute ischemic stroke due to carotid occlusions compared to smaller vessel occlusions have shown very poor results $^{9-12}$. Rubiera et al reported that patients with tandem occlusions were 3 times less likely to achieve recanalization as measured on follow-up by transcranial dopplers. ${ }^{3}$ In this setting, emergent carotid endarterectomy has been attempted as a method to recanalize the ICA, however results were similarly discouraging. ${ }^{2}$

The presence of a carotid occlusion may reflect an alteration of hemodynamic conditions and poor exposure to the thrombolytic agent deterring an effective response to systemic thrombolysis. ${ }^{13}$ Hemodynamically, carotid occlusions or near occlusions may contribute to blood stasis increasing the risk of distal restenosis or thrombosis after incomplete recanalization. Studies have shown that re-occlusion was four times more likely in patients with a proximal carotid and tandem distal occlusion when compared to the general acute ischemic stroke population. ${ }^{3}$

While it is controversial and not the standard of care, the use of endovascular interventions to recanalize ICA occlusions has recently been reported in several case reports.,6-8,14 Historically, the risk of traversing the occluded ICA had been thought to outweigh the benefits of thrombectomy due to dislodging more distal emboli and the risk of perforation or dissection. Lavallee et al reported their experience with endovascular stent-assisted thrombolysis in six patients. ${ }^{7}$ Three-month clinical followup showed neurological improvement when compared to a similar population receiving IV tPA only. Baumgartner et al reported their experience in four patients treated by carotid stenting and intra-arterial thrombolysis for ICA dissections causing tandem occlusions. ${ }^{8}$ They observed similar outcomes when compared to 14 similar patients treated with IV tPA alone. Mourand et al reported on two patients with internal carotid dissections causing tandem MCA occlusion treated by self-expandable/ 


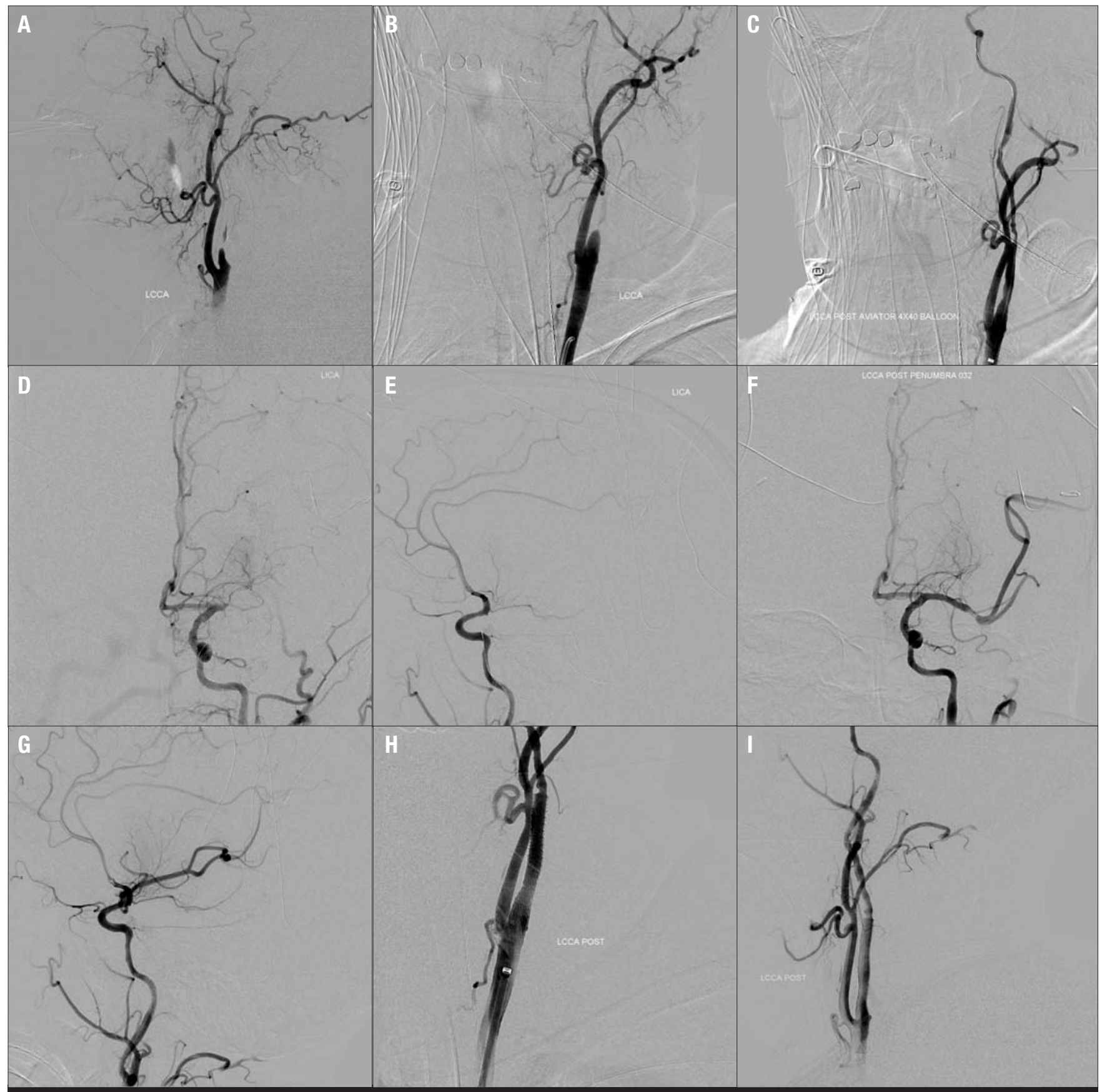

Figure 1

Cerebral angiograms showing proximal internal carotid artery occlusion thrombolysis and stenting, followed by tandem middle cerebral artery occlusion thrombolysis. $(A, B)$ digital subtraction angiogram in the working projection showing the fronral and lateral views of the occluded internal carotid artery. (C) Frontal DSA showing recanalization of the previously occluded vessel, following balloon. (D,E) Tandem middle cerebral artery (M1) occlusion. (F,G) DSA frontal and lateral projections showing successful recanalization of the MCA after balloon angioplasty and Penumbra retrieval. (H-I) DSA fronal and lateral projections showing final arterial runs of the internal carotid artery displaying sustained patency of the carotid circulation. 
stents and IA tPA with excellent outcomes at 1 year follow-up. ${ }^{6}$ Overall, these studies suggested this technique to be technically feasible with recanalization rates from $37-100 \%$ and clinical improvement of 53-94\%. ${ }^{5}$

In our institution, the goal of endovascular intervention is to restore blood flow to ischemic areas and decrease the extent of cerebral blood volume loss. Urgent recanalization of the ICA allows full access to tandem MCA thrombus for either chemical or mechanical thrombolysis. After recanalization, carotid stent placement also protects the vessel in the presence of an intimal tear, reducing the risk of re-occlusion and further embolic events. In our institution, revascularization of the carotid and intracranial circulations is achieved in approximately $89 \%$ of patients. Our clinical outcome shows improvement of all patient's NIHSS from an average of 15 on admission to 7 on discharge. While all patients are initially densely hemiplegic, an average of $50 \%$ are ambulatory on discharge.

\section{Conclusion}

In conclusion, an aggressive endovascular stroke protocol combining urgent carotid artery stenting with intracranial thrombolysis for acute internal carotid and tandem middle cerebral artery occlusions in the setting of ischemic stroke offers a feasible and efficacious intervention that compares favorably to the use of ivTPA.

\section{References}

1. Ducrocq X, Lacour JC, Debouverie M, Bracard S, Girard F, Weber M. Cerebral ischemic accidents in young subjects. A prospective study of 296 patients aged 16 to 45 years]. Rev Neurol (Paris). 1999;155:575-582.

2. Meyer FB, Sundt TM,Jr, Piepgras DG, Sandok BA, Forbes G. Emergency carotid endarterectomy for patients with acute carotid occlusion and profound neurological deficits. Ann Surg. 1986;203:82-89.

3. Rubiera M, Ribo M, Delgado-Mederos R, Santamarina E, Delgado P, Montaner J, Alvarez-Sabin J, Molina CA. Tandem internal carotid artery/middle cerebral artery occlusion: an independent predictor of poor outcome after systemic thrombolysis. Stroke. 2006;37:2301-2305.

4. Wang H, Lanzino G, Fraser K, Tracy P, Wang D. Urgent endovascular treatment of acute symptomatic occlusion of the cervical internal carotid artery. J Neurosurg. 2003;99:972-977.

5. Sugg RM, Malkoff MD, Noser EA, Shaltoni HM, Weir R, Cacayorin ED, Grotta JC. Endovascular recanalization of internal carotid artery occlusion in acute ischemic stroke. AJNR Am J Neuroradiol. 2005;26:2591-2594.

6. Mourand I, Brunel H, Vendrell JF, Thouvenot E, Bonafe A. Endovascular stent-assisted thrombolysis in acute occlusive carotid artery dissection. Neuroradiology. 2010;52:135-140.

7. Lavallee PC, Mazighi M, Saint-Maurice JP, Meseguer E, Abboud H, Klein IF, Houdart E, Amarenco P. Stent-assisted endovascular thrombolysis versus intravenous thrombolysis in internal carotid artery dissection with tandem internal carotid and middle cerebral artery occlusion. Stroke. 2007;38:2270-2274

8. Baumgartner RW, Georgiadis D, Nedeltchev K, Schroth G, Sarikaya H, Arnold M. Stent-assisted endovascular thrombolysis versus intravenous thrombolysis in internal carotid artery dissection with tandem internal carotid and middle cerebral artery occlusion. Stroke. 2008;39:e27-8.

9. Christou I, Felberg RA, Demchuk AM, Burgin WS, Malkoff M, Grotta JC, Alexandrov AV. Intravenous tissue plasminogen activator and flow improvement in acute ischemic stroke patients with internal carotid artery occlusion. J Neuroimaging. 2002;12:119-123.
10. Derex L, Nighoghossian N, Turjman F, Hermier M, Honnorat J, Neuschwander P, Froment JC, Trouillas P. Intravenous $\mathrm{PA}$ in acute ischemic stroke related to internal carotid artery dissection. Neurology. 2000;54:2159-2161.

11. Kim YS, Garami Z, Mikulik R, Molina CA, Alexandrov AV, CLOTBUST Collaborators. Early recanalization rates and clinical outcomes in patients with tandem internal carotid artery/middle cerebral artery occlusion and isolated middle cerebral artery occlusion. Stroke. 2005;36:869-871.

12. Linfante I, Llinas RH, Selim M, Chaves C, Kumar S, Parker RA, Caplan LR, Schlaug G. Clinical and vascular outcome in internal carotid artery versus middle cerebral artery occlusions after intravenous tissue plasminogen activator. Stroke. 2002;33:2066-2071.

13. Rubiera M, Alvarez-Sabin J, Ribo M, Montaner J, Santamarina E, Arenillas JF, Huertas R, Delgado P, Purroy F, Molina CA. Predictors of early arterial reocclusion after tissue plasminogen activator-induced recanalization in acute ischemic stroke. Stroke. 2005;36:1452-1456.

14. (14) Srinivasan A, Goyal M, Stys P, Sharma M, Lum C. Microcatheter navigation and thrombolysis in acute symptomatic cervical internal carotid occlusion. AJNR Am J Neuroradiol. 2006;27:774-779.

\section{Corresponding Author}

Stavropoula I. Tjoumakaris, MD

Department of Cerebrovascular and

Endovascular Neurosurgery

Thomas Jefferson University Hospital 909 Walnut St, 2nd Floor

Philadelphia, PA 19107

Email: stavropoula.tjoumakaris@jefferson.edu Phone: 215-503-3383

\section{Brain power}

\section{Jefferson. \\ Hospital for Neuroscience}

1-800-JEFF-NOW

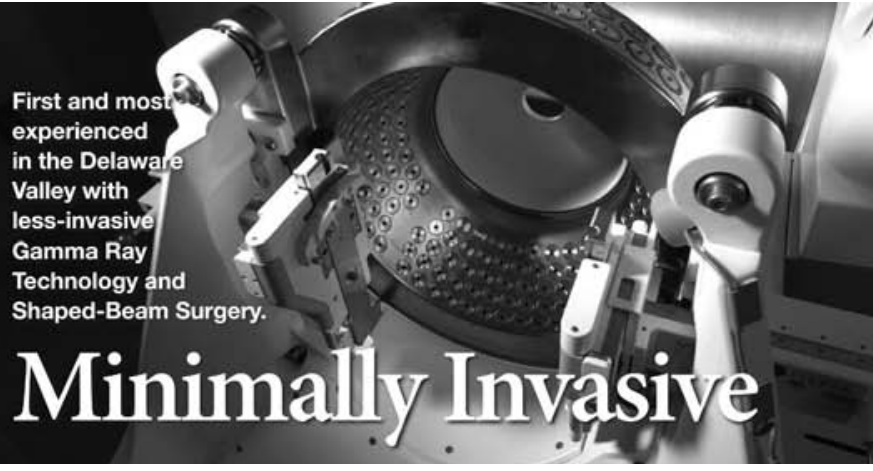

